\title{
Significance of Small Renal Artery Lesions in Patients with Antineutrophil Cytoplasmic Antibody-associated Glomerulonephritis
}

\author{
Akiko Endo, Junichi Hoshino, Tatsuya Suwabe, Keiichi Sumida, Koki Mise, Rikako Hiramatsu, \\ Eiko Hasegawa, Masayuki Yamanouchi, Noriko Hayami, Naoki Sawa, Kenmei Takaichi, \\ Kenichi Ohashi, Takeshi Fujii, and Yoshifumi Ubara
}

\begin{abstract}
Objective. Antineutrophil cytoplasmic antibody (ANCA)-associated glomerulonephritis is a vasculitis affecting the glomerular capillaries and small renal arteries. Although crescent formation has been reported to be characteristic of this condition, the significance of coexisting vasculitis affecting the small renal arteries has not been investigated.

Methods. Fifty patients with ANCA-positive rapidly progressive glomerulonephritis whose renal biopsy specimens contained arterioles and/or interlobular arteries were retrospectively evaluated. Cellular crescents and/or necrotizing glomerulonephritis were noted in all 50 patients. Ten patients had vasculitis of the small renal arteries (group A) and 40 patients were without such vasculitis (group B). The clinical features of these 2 groups were compared.

Results. Group A comprised 4 patients who had granulomatosis with polyangiitis (GPA) and 6 with microscopic polyangiitis (MPA), while group B included 1 patient with GPA and 39 with MPA. No patient in either group had eosinophilic granulomatosis with polyangiitis. The C-reactive protein (CRP) level was significantly higher in group A compared with group B (11.58 \pm 6.19 vs $2.7 \pm 3.55$ $\mathrm{mg} / \mathrm{dl}, \mathrm{p}<0.05$ ), and pulmonary involvement was more frequent in group A than group B (80\% vs $37.5 \%, \mathrm{p}<0.05)$.

Conclusion. In patients with ANCA-positive glomerulonephritis, vasculitis of small renal arteries may be associated with systemic vasculitis (including pulmonary involvement) because of elevated CRP, a systemic inflammatory marker related to overproduction of interleukin 6. (First Release April 15 2014; J Rheumatol 2014;41:1140-6; doi:10.3899/jrheum.130657)
\end{abstract}

Key Indexing Terms:

VASCULITIS OF SMALL RENAL ARTERIES

GLOMERULONEPHRITIS ANTINEUTROPHIL CYTOPLASMIC ANTIBODIES

The classification of vasculitis was developed by the first international Chapel Hill consensus conference in 1994 $(\mathrm{CHCC} 1994)^{1}$. Combining the American College of Rheumatology criteria $^{2}$ and the Lanham criteria for Churg-Strauss syndrome ${ }^{3}$, an algorithm for differential

From the Nephrology Center and Okinaka Memorial Institute for Medical Research, Department of Pathology, and Nephrology Center, Toranomon Hospital; Division of Nephrology, Department of Internal Medicine, Kyorin University School of Medicine, Tokyo, Japan.

Funded by the Okinaka Memorial Institute for Medical Research.

A. Endo, MD, Nephrology Center, Toranomon Hospital, and Division of Nephrology, Department of Internal Medicine, Kyorin University School of Medicine; J. Hoshino, MD; T. Suwabe, MD; K. Sumida, MD; K. Mise, MD; R. Hiramatsu, MD; E. Hasegawa, MD; M. Yamanouchi, MD;

N. Hayami, MD; N. Sawa, MD, Nephrology Center, Toranomon Hospital; $K$. Takaichi, MD, Nephrology Center and Okinaka Memorial Institute for Medical Research; K. Ohashi, MD, Nephrology Center; T. Fujii, MD, Department of Pathology; Y. Ubara, MD, Nephrology Center and Okinaka Memorial Institute for Medical Research, Toranomon Hospital. Address correspondence to Dr. Endo, Yoshifumi Ubara, Nephrology Center, Toranomon Hospital, 2-2-2 Toranomon Minato-ku, Tokyo, Japan 105-8470.E-mail: kokia5n@yahoo.co.jp, and ubara@toranomon.gr.jp Full Release Article. For details see Reprints/Permissions at jrheum.org Accepted for publication February 4, 2014. diagnosis was proposed by the European Medicines Agency in $2007^{4}$. This classification was updated by the second international CHCC in $2012^{5,6}$. At that time, small vessel vasculitis was divided into antineutrophil cytoplasmic antibody (ANCA)-associated vasculitis (AAV) and immune complex-type vasculitis. The former type comprises microscopic polyangiitis (MPA), granulomatosis with polyangiitis (GPA), and eosinophilic granulomatosis with polyangiitis (Churg-Strauss syndrome; EGPA). Renal involvement in patients with AAV (ANCA-associated glomerulonephritis) features vasculitis that affects both the glomerular capillaries and the small arteries, including the arterioles and the interlobular and arcuate arteries. However, previous studies of ANCA-associated glomerulonephritis have largely focused on glomerular capillary lesions, such as necrotizing glomerulonephritis with crescent formation, and the significance of small artery vasculitis has not been investigated in much detail. In fact, only a few studies have assessed vasculitis of the small renal arteries $7,8,9,10,11$. Accordingly, we performed a retrospective investigation to determine whether the presence of small artery vasculitis had a clinical effect in patients with ANCA-associated glomerulonephritis.

Personal non-commercial use only. The Journal of Rheumatology Copyright @ 2014 . All rights reserved. 


\section{MATERIALS AND METHODS}

Patients. Sixty-one patients were diagnosed by renal biopsy as having ANCA-positive glomerulonephritis at our hospital from 1991 to 2011. Eleven patients were excluded because they had secondary vasculitis such as lupus vasculitis, antiglomerular basement membrane disease, or biopsy errors (specimens without cellular crescents and/or necrotizing glomerulonephritis and small arteries). The remaining 50 patients with ANCA-associated glomerulonephritis were enrolled in this retrospective study. They were divided into group A (with concomitant vasculitis of small arteries including the arterioles, interlobular arteries, or arcuate arteries) and group B (without small artery vasculitis), and various clinical characteristics were compared between the 2 groups (Table 1).

Classification of vasculitis. According to the European Medicines Agency algorithm ${ }^{4}$, the patients were divided into 3 subgroups: EGPA, GPA, and MPA, including renal-limited AAV (RLV; defined as no evidence of vasculitis outside the kidneys).

Measurement of ANCA. ANCA was measured at the time of presentation before renal biopsy. The first 3 patients were positive for perinuclear (p)ANCA or cytoplasmic (c)ANCA by indirect immunofluorescence, while the remaining 47 patients were positive for myeloperoxidase (MPO) ANCA or proteinase 3 (PR3)-ANCA by ELISA (Nipro).

Histological evaluation of renal biopsy specimens. Renal biopsy specimens were fixed in $6 \%$ formalin, embedded in paraffin, cut into 1-2 $\mu \mathrm{m}$ sections, and stained with H\&E, periodic acid Schiff, Weigert's elastica-van Gieson, Masson trichrome, periodic acid methenamine silver (PAM), or PAM-Masson stain for light microscopy. All 50 renal biopsy specimens were reviewed by at least 2 pathologists. A cellular crescent was defined as a lesion consisting of proliferative epithelial cells and inflammatory cells that occupied $25 \%$ or more of Bowman's space, and included at least 2 layers of proliferating cells (Figure 1A). Vasculitis of the small arteries was defined as fibrinoid necrosis (red on Masson trichrome staining and/or H\&E staining) accompanied by infiltration of leukocytes (Figure 1B).

Treatment. Patients were treated with various regimens. Nineteen patients received oral prednisolone (PSL; $0.8 \mathrm{mg} / \mathrm{kg}$ ) with concomitant intravenous methylprednisolone pulse therapy $(500 \mathrm{mg} /$ day for 3 days). Thereafter, cyclophosphamide was added for 6 patients. Among the other 31 patients, only oral PSL was given to 4 patients, while 3 patients received oral PSL with oral cyclophosphamide and 24 patients received oral PSL with intravenous cyclophosphamide ( $500 \mathrm{mg} /$ day for 3 days).

Relapse. Relapse was defined as the clinical requirement for an increase in the dose of PSL because of deterioration of renal function with nephritic sediment or symptoms of vasculitis.

Statistical analysis. Data were summarized as proportions or as the mean $( \pm$ SD). Categorical variables were analyzed with the chi-square test or Fisher's exact test as appropriate, while continuous variables were

Table 1A. Clinical and laboratory findings for group A.

\begin{tabular}{|c|c|c|c|c|c|c|c|c|c|c|c|c|c|c|c|c|c|}
\hline Case & $\begin{array}{l}\text { Age, } \\
\text { yrs }\end{array}$ & Sex & $\begin{array}{c}\text { ANCA } \\
\text { (EU) }\end{array}$ & Diagnosis & $\begin{array}{c}\text { CRP, } \\
\mathrm{mg} / \\
\mathrm{dl}\end{array}$ & $\begin{array}{c}\mathrm{Cr}, \\
\mathrm{mg} / \\
\mathrm{dl}\end{array}$ & $\begin{array}{l}\text { Hematuria, } \\
\text { grade }\end{array}$ & $\begin{array}{l}\text { Proteinuria } \\
\text { g/day }\end{array}$ & $\begin{array}{c}\text { Crescents } \\
(\%)\end{array}$ & $\begin{array}{c}\text { Global } \\
\text { Sclerosis } \\
(\%)\end{array}$ & Vasculitis & $\begin{array}{c}\text { Organ } \\
\text { Involvement } \\
\text { (extrarenal) }\end{array}$ & $\begin{array}{l}\text { Initial } \\
\text { Therapy }\end{array}$ & $\begin{array}{l}\text { Maintenance } \\
\text { Therapy }\end{array}$ & $\begin{array}{l}\text { Disease } \\
\text { Duration } \\
\text { (mos) }\end{array}$ & Relapse & $\begin{array}{l}\text { End of } \\
\text { Followup }\end{array}$ \\
\hline 2 & 84 & F & $\begin{array}{l}\text { MPO } \\
297\end{array}$ & MPA & 2.7 & 8.7 & 4 & 1.62 & 67 & 0 & Interlobular & $(-)$ & $\begin{array}{l}\text { PSL } \\
\text { CYC }\end{array}$ & & 2 & 0 & $\begin{array}{c}\text { Death } \\
\text { (renal } \\
\text { failure) }\end{array}$ \\
\hline 4 & 80 & M & $\begin{array}{c}\text { MPO } \\
78\end{array}$ & MPA & 12.1 & 3.3 & 2 & 0.88 & 56 & 20 & $\begin{array}{c}\text { Arteriole } \\
\text { Interlobular }\end{array}$ & ILD & $\begin{array}{l}\text { mPSL pulse } \\
\text { PSL }\end{array}$ & PSL & 25 & 0 & Cr 2.9 \\
\hline 5 & 58 & F & $\begin{array}{l}\text { MPO } \\
288\end{array}$ & MPA & 13.4 & 1 & 3 & 0.73 & 58 & 8 & $\begin{array}{l}\text { Interlobular } \\
\text { Arcuate }\end{array}$ & ILD & $\begin{array}{l}\text { mPSL pulse } \\
\text { PSL }\end{array}$ & PSL & 20 & 0 & Cr 1.1 \\
\hline 6 & 70 & F & $\begin{array}{c}\text { MPO } \\
88\end{array}$ & MPA & 22.1 & 9.1 & 5 & 0.89 & 79 & 4 & & $\begin{array}{l}\text { Pulmonary } \\
\text { hemorrhage }\end{array}$ & mPSL pulse & PSL & 19 & 0 & Cr 2.1 \\
\hline 7 & 68 & F & $\mathrm{P}(+)$ & GPA & 11.9 & 1.1 & 4 & 0.5 & 0 & 40 & $\begin{array}{l}\text { Arteriole } \\
\text { Interlobular }\end{array}$ & ILD & PSL & PSL & 163 & 0 & $\begin{array}{c}\text { Death } \\
\text { (Intestinal } \\
\text { perforation) }\end{array}$ \\
\hline 8 & 57 & F & PR3 10 & GPA & 19.5 & 6.7 & 5 & 1.95 & 70 & 10 & Interlobular & $\begin{array}{c}\text { Pulmonary } \\
\text { tumor } \\
\text { Neuritis }\end{array}$ & $\begin{array}{l}\text { mPSL pulse } \\
\text { PSL, CYC }\end{array}$ & PSL & 50 & 3 & Hemodialysis \\
\hline 9 & 66 & $\mathrm{M}$ & $\begin{array}{c}\text { MPO } \\
359\end{array}$ & GPA & 5.7 & 0.6 & 4 & 0.13 & 9 & 12 & Interlobular & $\begin{array}{c}\text { ILD } \\
\text { Neuritis }\end{array}$ & $\begin{array}{l}\text { mPSL pulse } \\
\text { PSL, CYC }\end{array}$ & $\begin{array}{l}\text { PSL } \\
\text { MZB }\end{array}$ & 60 & 1 & Cr 1.8 \\
\hline \multirow[t]{2}{*}{10} & 62 & M & $\begin{array}{c}\text { PR3 } \\
130\end{array}$ & GPA & 14.32 & 4 & 5 & 1.66 & 2 & 45 & Arteriole & $\begin{array}{l}\text { Pulmonary } \\
\text { tumor }\end{array}$ & mPSL pulse & PSL & 6 & 0 & $\operatorname{Cr} 2.6$ \\
\hline & & & & & & & & & & & Interlobular & $\begin{array}{l}\text { Celebral } \\
\text { infarction }\end{array}$ & PSL, CYC & & & & \\
\hline
\end{tabular}

Crescents: the percentage of glomeruli with crescents. ILD: interstitial lung disease; PSL: prednisonone; mPLSpulse: intravenous methylprednisolone pulse therapy; CYC: cyclophosphamide; MZB: mizorbine; GPA: granulomatosis with polyangiitis; EU: ELISA unit; CRP: C-reactive protein; Cr: creatinine; MPO: myeloperoxidase; MPA: microscopic polyangiitis; PR3: proteinase 3. 
Table 1B. Clinical and laboratory findings for group A.

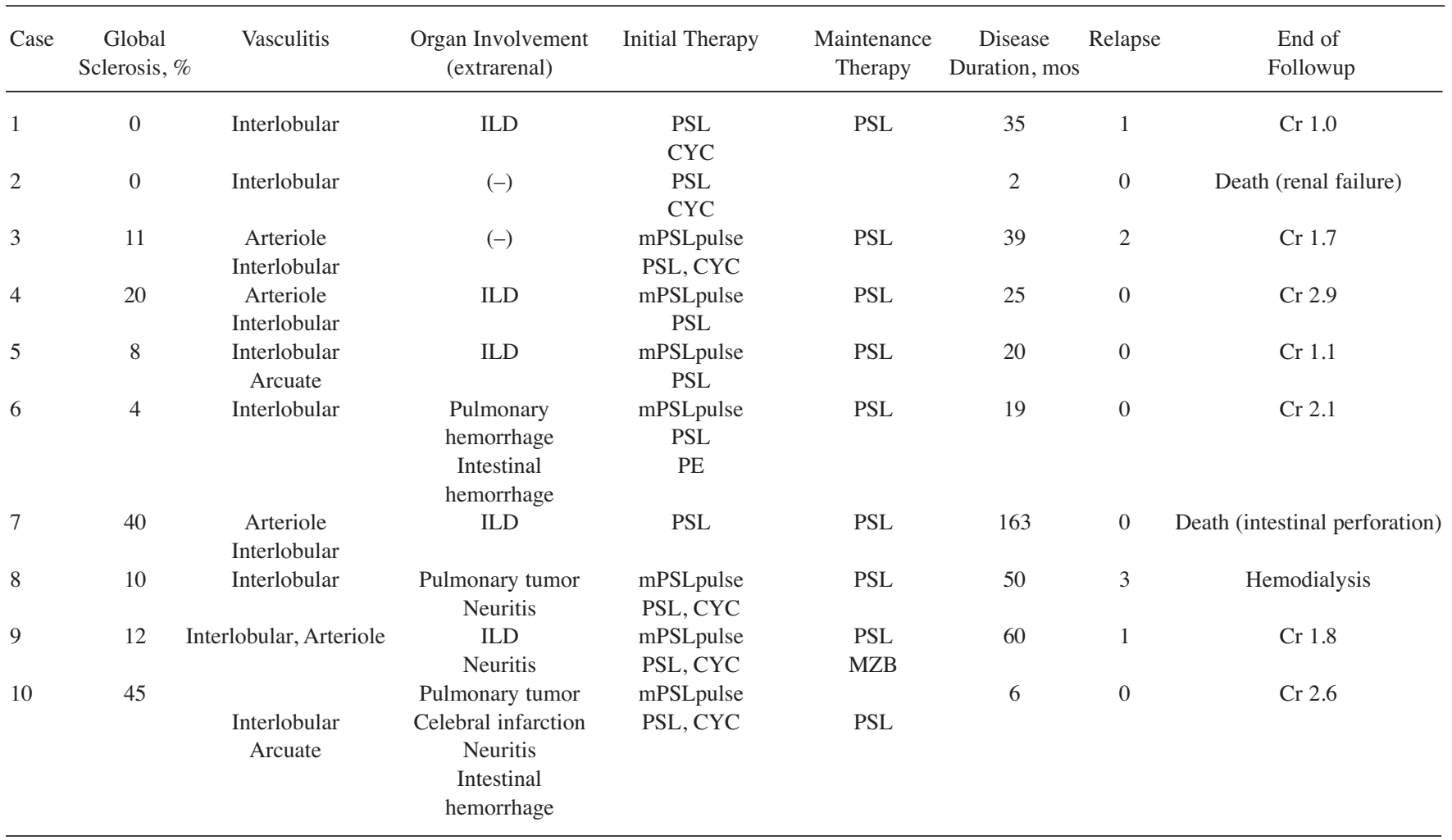

GPA was diagnosed by the following findings: Case 7 : histological granulomatous inflammation of the renal interstitium; Case 8: pulmonary nodules; Case 9: histological granulomatous inflammation in the lungs; Case 10: chronic sinusitis and pulmonary nodules. Hematuria: grade 1, < 1/high power field (HPF); grade 2, 1 to 5/HPF; grade 3, 6 to 10/HPF; grade 4, 11 to 30/HPF; grade 5, > 30/HPF. Crescents: the percentage of glomeruli with crescents. ILD: interstitial lung disease; PSL: prednisonone; mPLSpulse: intravenous methylprednisolone pulse therapy; CYC: cyclophosphamide; PE: plasma exchange; MZB: mizorbine; ANCA: antineutrophil cytoplasmic antibody; GPA: granulomatosis with polyangiitis; CRP: C-reactive protein; Cr: creatinine; MPO: myeloperoxidase; MPA: microscopic polyangiitis; PR3: proteinase 3.

compared using ANOVA. The Mann-Whitney U test was used to compare nonparametric variables between 2 groups. Cumulative event rates were estimated by Kaplan-Meier survival analysis, and were compared using the $\log$-rank test. A p value of $<0.05$ was considered to indicate statistical significance. All analyses were performed using SPSS software (version $20.0)$.

\section{RESULTS}

Patient characteristics and laboratory data. Among the 50 patients with ANCA-associated glomerulonephritis, 10 patients were classified into group A and the other 40 patients into group B (Table 1). Group A comprised 4 patients $(40 \%)$ with GPA and 6 patients with MPA. Two of the latter 6 patients had RLV. Group B included 1 patient with GPA and 39 patients with MPA. Among the latter 39 patients, 21 patients had RLV. None of the patients in either group had EGPA.

The CRP level was significantly higher in group A compared with group B, being $11.58 \pm 6.19$ versus $2.7 \pm$ $3.55 \mathrm{mg} / \mathrm{dl}$, respectively $(\mathrm{p}<0.01)$. There were no significant differences in the age, sex, serum creatinine, MPO, and PR3-ANCA titers, or proteinuria or hematuria between the 2 groups (Table 2). Pulmonary involvement showed a signifi- cant difference in prevalence between group A and group B, being found in 9 patients $(80 \%)$ and 15 patients $(37.5 \%)$, respectively $(\mathrm{p}<0.05)$. In group $\mathrm{A}$, the breakdown of pulmonary involvement was pulmonary hemorrhage in 1 patient, interstitial lung disease in 5 patients, and pulmonary nodules in 2 patients, while group B had pulmonary hemorrhage in 5 patients and interstitial lung disease in 10 patients.

Glomerular changes. There were no significant differences between the 2 groups in the percentage of glomeruli with crescent formation and fibrinoid necrosis. However, the percentage of glomeruli with global sclerosis was lower in group A than in group B $(15.0 \pm 15.7 \%$ vs $35.0 \pm 29.1 \%)$. When the number of glomeruli per specimen (as a surrogate for the amount of cortex sampled) was compared between groups A and group B, there was no significant difference $(27.0 \pm 19.6$ and $23.2 \pm 14.4$, respectively). This finding suggested that the number of arteries affected by vasculitis was not correlated with the number of affected glomeruli including small arteries. Vasculitis was not detected in the medullary region.

Prognosis. The relapse rate was significantly higher in 


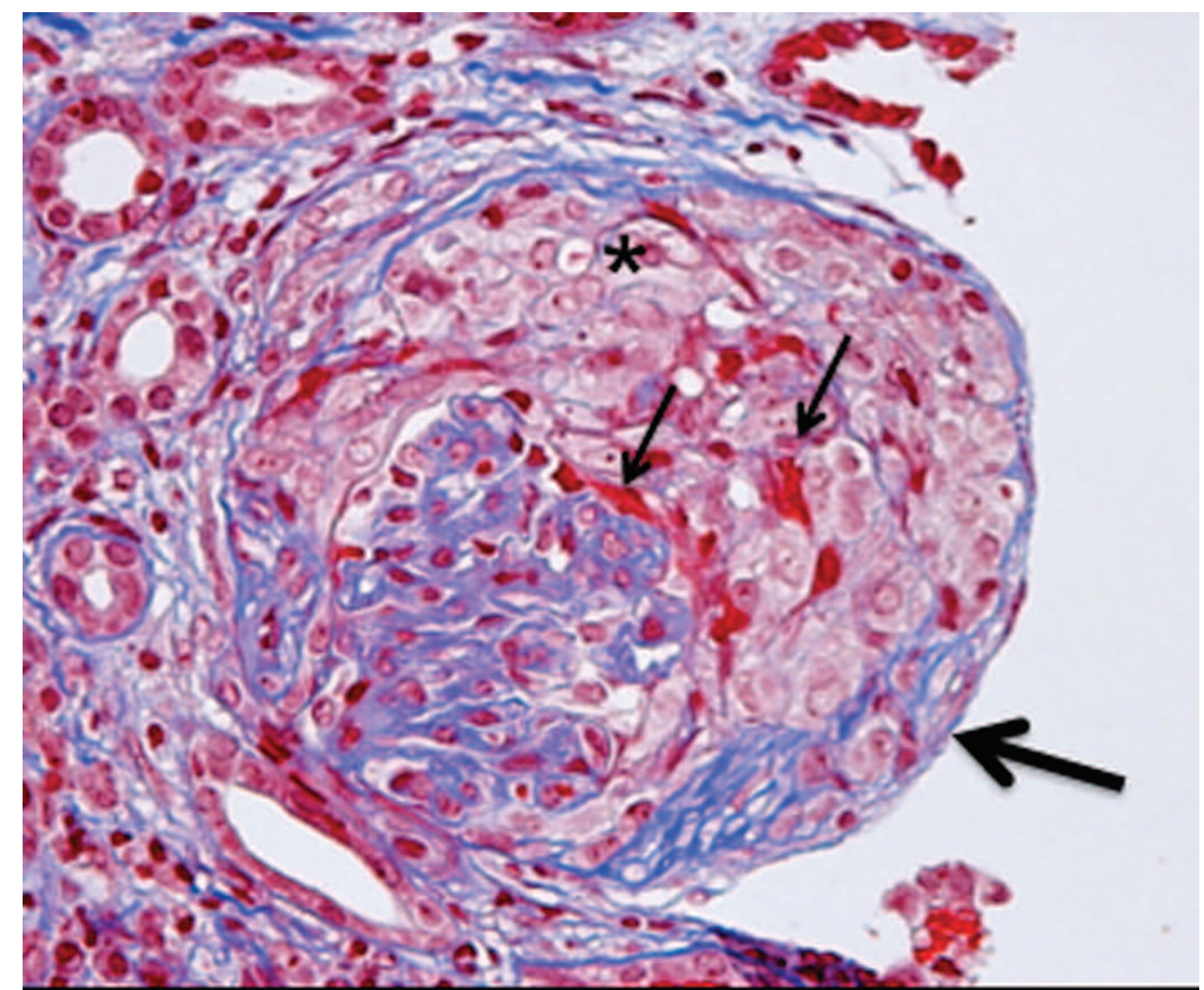

A

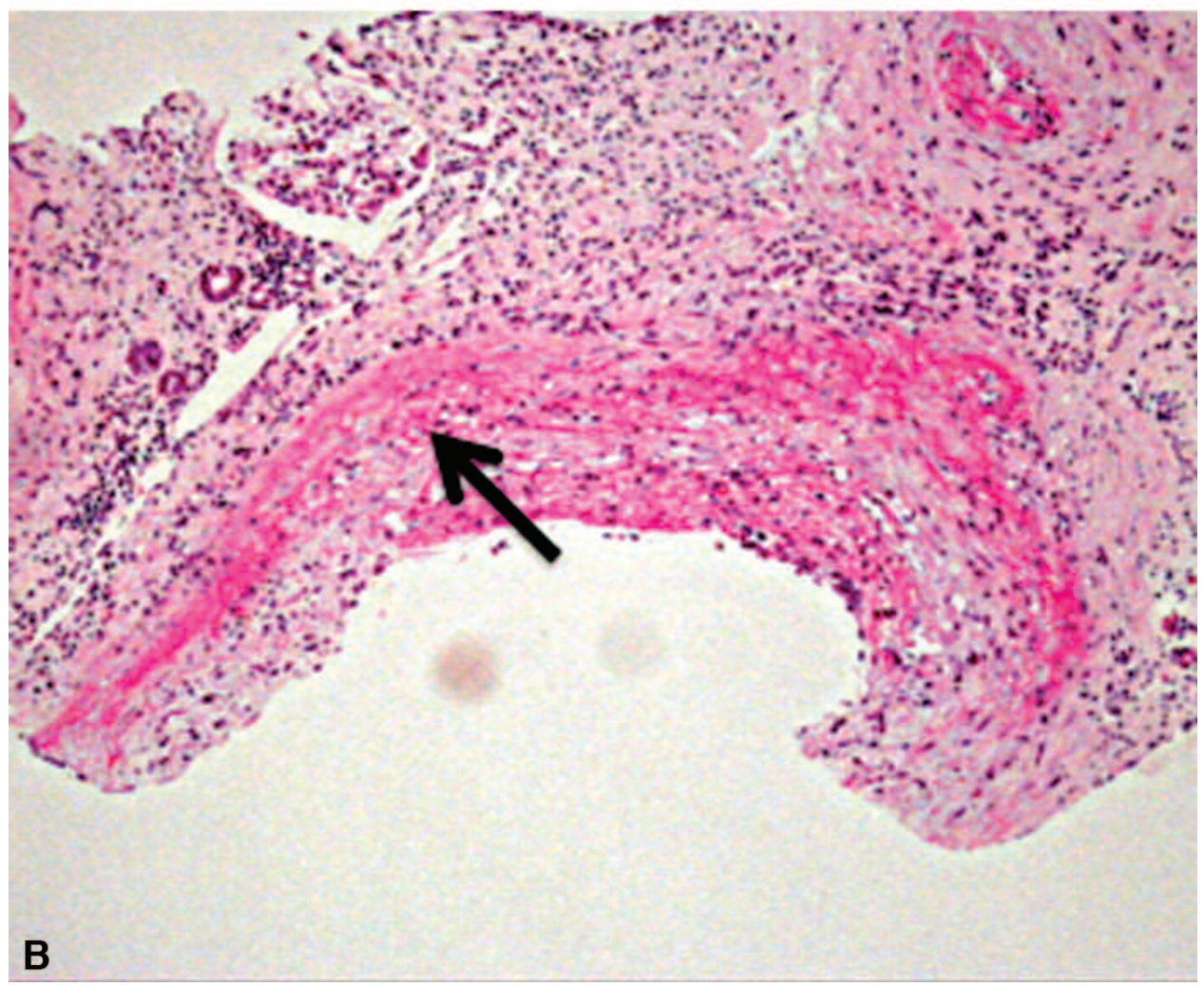

Figure 1. A. Periodic acid methenamine silver-Masson staining shows a cellular crescent $(*)$ with fibrin-rich material (small arrows) and fibrillary material (large arrow). B. H\&E stain shows fibrinoid necrosis extending from the interlobular artery to arcuate artery. 
Table 2. Comparison of clinical findings and renal history between groups A and B. Data are $\mathrm{n}(\%)$ or mean \pm SD; Fisher's exact test, chi-square test, Mann-Whitney U test.

\begin{tabular}{lccc}
\hline Characteristics & Group A, $\mathrm{n}=10$ & Group B, $\mathrm{n}=40$ & $\mathrm{p}$ \\
\hline Age, yrs & $67.6 \pm 9.05$ & $61.4 \pm 15.5$ & $\mathrm{NS}$ \\
Sex, men/women & $3 / 7$ & $11 / 29$ & $\mathrm{NS}$ \\
Duration of disease, mos & $57.7 \pm 57.6$ & $78.8 \pm 63.0$ & $\mathrm{NS}$ \\
MPO-ANCA positive & $8(80)$ & $42(95.4)$ & $\mathrm{NS}$ \\
PR3-ANCA positive & $2(20)$ & $3(6.7)$ & $\mathrm{NS}$ \\
MPO-ANCA (EU) & $377.0 \pm 294.1$ & $272.0 \pm 336.7$ & $\mathrm{NS}$ \\
PR3-ANCA (EU) & $70.0 \pm 84.9$ & $59.3 \pm 51.9$ & $<0.01$ \\
C-reactive protein, mg/dl & $11.58 \pm 6.19$ & $2.7 \pm 3.55$ & $\mathrm{NS}$ \\
Creatinine (mg/dl) & $3.8 \pm 3.2$ & $3.7 \pm 3.0$ & $\mathrm{NS}$ \\
Urine protein, g/day & $0.94 \pm 0.65$ & $2.08 \pm 2.25$ & $\mathrm{NS}$ \\
Hematuria, $>$ 6/HPF & $9(90)$ & $40(100)$ & $\mathrm{NS}$ \\
Glomerular lesions; crescents $(\%)$ & $41.1 \pm 29.6$ & $42.9 \pm 29.4$ & 0.042 \\
Glomerular sclerosis $(\%)$ & $15.0 \pm 15.7$ & $35.0 \pm 29.1$ & $\mathrm{NS}$ \\
Fibrinoid necrosis & $6(60)$ & $16(40)$ & 0.016 \\
Pulmonary involvement & $8(80)$ & $15(37.5)$ & $\mathrm{NS}$ \\
Others (neuron, ear, skin) & $4(40)$ & $7(17.5)$ & $<(2.5)$ \\
GPA (patients) & $4(40)$ & $\mathrm{n}=39(\mathrm{n}=21)$ & - \\
MPA (RLV) & $\mathrm{n}=6(\mathrm{n}=2)$ & &
\end{tabular}

HPF: high-power field; MPO: myeloperoxidase; ANCA: antineutrophil cytoplasmic antibody; PR3: proteinase 3; GPA: granulomatosis with polyangiitis; MPA: microscopic polyangiitis; RLV: renal-limited AAV; AAV: ANCA-associated vasculitis; NS: not significant.

group A than in group B ( $p<0.05$; Figure $2 \mathrm{~A})$. However, the renal survival rate (interval from the date of diagnosis to the occurrence of endstage renal failure requiring dialysis) did not show a significant difference between the 2 groups (Figure 2B), and the survival rate of the patients also showed no significant difference (Figure 2C). However, these findings should be interpreted with caution because the subjects received treatment by various regimens.

\section{DISCUSSION}

Among many studies of ANCA-related glomerulonephritis, vasculitis of the small arteries, including interstitial capillaries, arterioles, interlobular arteries, and arcuate arteries, has been reported by several authors. Bajema, et al performed a metaanalysis of 349 patients with Wegener's granulomatosis (now called GPA) reported in the literature from 1979 to $1997^{7}$, including evaluation of data on 134 renal biopsies. The most frequent lesion revealed by renal biopsy was extracapillary proliferation $(70 \%)$, followed by fibrinoid necrosis of the glomerular tufts (54\%). Vasculitis of the interstitial arteries and arterioles was present in almost $20 \%$, whereas renal granulomas were seen in only 7 biopsy specimens. However, the details of vasculitis of the small arteries were not analyzed. In addition, Hauer, et al analyzed 173 patients with MPA $(n=80)$, GPA $(n=73)$, or renal-limited vasculitis $(n=19)^{8}$. Glomerular lesions (fibrinoid necrosis of the glomeruli, crescents, glomerulosclerosis, periglomerular infiltrates, and granulomatous reaction) and tubulointerstitial lesions (interstitial infiltrates, interstitial fibrosis, and tubular atrophy) were evaluated.
They reported that $12 \%$ of the patients had interstitial vasculitis, but we could not determine whether such interstitial vasculitis corresponded to vasculitis of the small renal arteries from the description in their article. Further, Chen, et al evaluated renal biopsies from 61 patients with GPA and 44 patients with $\mathrm{MPA}^{9}$, focusing on vascular lesions. Among the 39 patients with MPO-ANCA-positive GPA, necrotizing lesions of interlobular arteries were found in 4 $(10.3 \%)$. Among the 22 patients with PR3-ANCA-positive GPA, necrotizing lesions of the arterioles were found in only 1 case. Out of the 44 patients with MPO-ANCA-positive MPA, 2 had necrotizing lesions of the arterioles and another 2 had necrotizing lesions of the interlobular arteries. However, the characteristics of these 9 cases with small artery vasculitis were not reported.

Vizjak, et al reported on the prevalence of vasculitis in biopsies from patients with ANCA-associated glomerulonephritis. Active extraglomerular vasculitis was observed in $22.2 \%$, while chronic vascular lesions indicative of previous vasculitis were present in $11.9 \%{ }^{10}$. Extraglomerular renal vasculitis was identified in $38.3 \%$ of the patients with systemic vasculitis and in $10.0 \%$ of the patients with renal-limited vasculitis. They hypothesized that extraglomerular vasculitis of small renal vessel may be a predictor of systemic involvement, although they did not find any difference between PR3-positive patients and MPO-positive patients. The report by Yamagata, et al on Japanese patients with AAV may be useful for assessing the true value of the present study ${ }^{11}$. They investigated 46 patients with GPA, 344 patients with MPA, and 745 patients

Personal non-commercial use only. The Journal of Rheumatology Copyright @ 2014 . All rights reserved. 


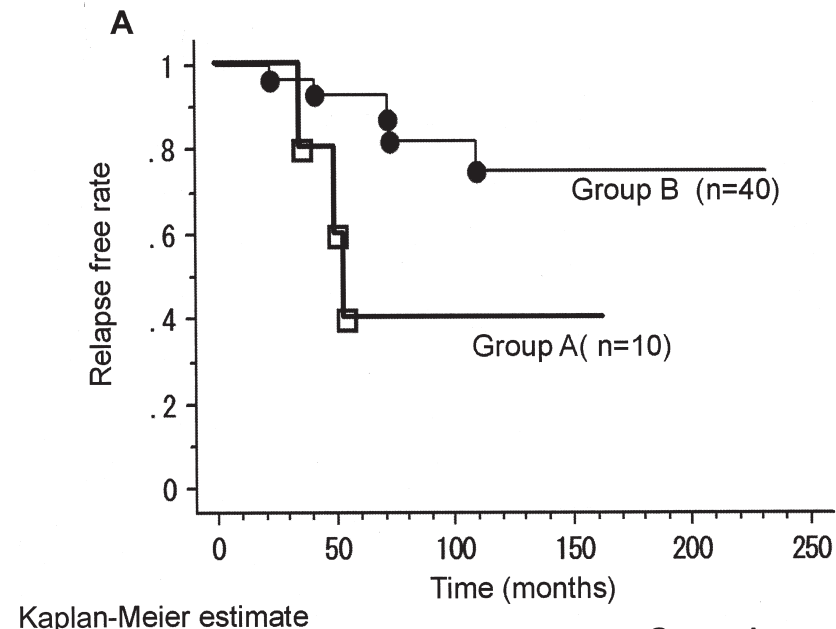

Kaplan-Meier estimate

Group A vs group B: $P=0.04$

by Log-rank.

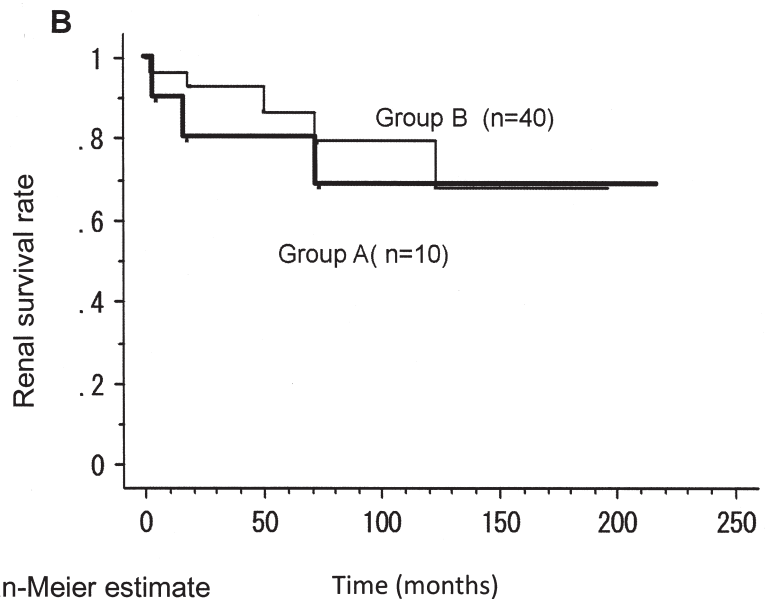

Kaplan-Meier estimate Time (months)

Group A vs group B: $\mathrm{P}=0.66$

by Log-rank.

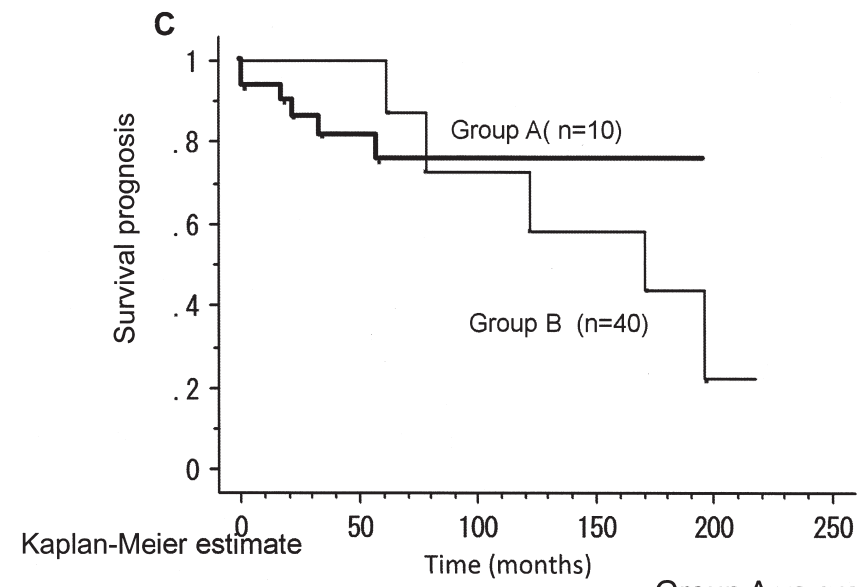

Group A vs group B: $P=0.12$

by Log-rank.

Figure 2. A. The relapse rate was significantly higher in group A than in group B $(\mathrm{p}<0.05$; group A vs group $\mathrm{B}: \mathrm{p}=0.04$ by log-rank). B. The renal survival rate did not show a significant difference between the 2 groups (group A vs group B: $\mathrm{p}=0.66$ by $\log$-rank). C. The prognosis also showed no significant difference between the 2 groups (group A vs group B: $p=0.12$ by log-rank). 
with RLV. Among the 745 patients with RLV, 656 patients (88.1\%) had MPO and 55 patients (7.4\%) had PR3. In addition, there were 316 patients $(91.8 \%)$ who had MPO and 21 patients $(6.1 \%)$ who had PR3 among the 344 patients with MPA, while 10 patients $(22.7 \%)$ had MPO and 33 patients (71.1\%) had PR3 among the 46 patients with GPA. It should be noted that the number of patients with GPA and/or PR3 is smaller in Japan than in Western countries.

Finally, Inoue, et al clarified the 3-dimensional morphology of the renal interlobular arteries in 6 autopsy cases of acute inflammatory MPA with necrotizing vasculitis by examination of serial paraffin sections ${ }^{12}$. All 19 lesions that were detected had microaneurysms, of which 18 were sausage-shaped and 1 was saccular. A possibility of a risk of rupture was suggested in patients with microaneurysms.

Our study showed that small renal artery lesions are closely related to elevation of CRP and pulmonary involvement. Because CRP is a systemic inflammatory marker that indicates the overproduction of interleukin 6, elevation of CRP may be involved in the process by which inflammation progresses from intraglomerular capillaries to small renal arteries and/or small systemic vessels, including the pulmonary arteries. This hypothesis is supported by the findings of Vizjak, et al ${ }^{10}$.

\section{ACKNOWLEDGMENT}

We thank Dr. Akira Yamada (Division of Nephrology, Department of Internal Medicine, Kyorin University School of Medicine) for his helpful advice.

\section{REFERENCES}

1. Jennette JC, Falk RJ, Andrassy K, Bacon PA, Churg J, Gross WL, et al. Nomenclature of systemic vasculitides. Proposal of an international consensus conference. Arthritis Rheum 1994; 37:187-92.

2. Fries JF, Hunder GG, Bloch DA, Michel BA, Arend WP, Calabrese LH, et al. The American College of Rheumatology 1990 criteria for the classification of vasculitis. Summary. Arthritis Rheum 1990;33:1135-6.
3. Lanham JG, Elkon KB, Pusey CD, Hughes GR. Systemic vasculitis in asthma and eosinophilia: a clinical approach to the Churg Strauss Syndrome. Medicine 1984;63:65-81.

4. Watts R, Lane S, Hanslik T, Hauser T, Hellmich B, Koldingsnes W, et al. Development and validation of a consensus methodology for the classification of the ANCA-associated vasculitides and polyarteritis nodosa for epidemiological studies. Ann Rheum Dis 2007;66:222-7.

5. Falk RJ, Gross WL, Guillevin L, Hoffman GS, Jayne DR, Jennette JC, et al; American College of Rheumatology; American Society of Nephrology; European League Against Rheumatism.

Granulomatosis with polyangiitis (Wegener's): an alternative name for Wegener's granulomatosis. Arthritis Rheum 2011;63:863-4.

6. Jennette JC, Falk RJ, Bacon PA, Basu N, Cid MC, Ferrario F, et al. 2012 Revised International Chapel Hill Consensus Conference nomenclature of vasculitides. Arthritis Rheum 2013;65:1-11.

7. Bajema IM, Hagen EC, van der Woude FJ, Bruijn JA. Wegener's granulomatosis: a meta-analysis of 349 literary case reports. J Lab Clin Med 1997;129:17-22.

8. Hauer HA, Bajema IM, van Houwelingen HC, Ferrario F, Noël LH, Waldherr R, et al; European Vasculitis Study Group (EUVAS). Renal histology in ANCA-associated vasculitis: differences between diagnostic and serologic subgroups. Kidney Int 2002;61:80-9.

9. Chen M, Yu F, Wang SX, Zou WZ, Zhang Y, Zhao MH, et al. Renal histology in Chinese patients with anti-myeloperoxidase autoantibody-positive Wegener's granulomatosis. Nephrol Dial Transplant 2007;22:139-45.

10. Vizjak A, Rott T, Koselj-Kajtna M, Rozman B, Kaplan-Pavlovcic S, Ferluga D. Histologic and immunohistologic study and clinical presentation of ANCA-associated glomerulonephritis with correlation to ANCA antigen specificity. Am J Kidney Dis 2003; 41:539-49.

11. Yamagata K, Usui J, Saito C, Yamaguchi N, Hirayama K, Mase K, et al. ANCA-associated systemic vasculitis in Japan: clinical features and prognostic changes. Clin Exp Nephrol 2012;16:580-8.

12. Inoue M, Akikusa B, Masuda Y, Kondo Y. Demonstration of microaneurysms at the interlobular arteries of the kidneys in microscopic polyangiitis: a three-dimensional study. Hum Pathol 1998;29:223-7. 